\title{
Resonance evaluation of Gadolinium isotopes
}

\author{
Luiz Leal ${ }^{1 *}$, Vladimir Sobes $^{2}$, Federico Rocchi ${ }^{3}$, Danila Roubtsov ${ }^{4}$, Jimmy Chow $^{4}$, and Cristian \\ Massimi $^{5}$ \\ ${ }^{1}$ Institut de Radioprotection et de Sûreté Nucléarie, Fontenay-aux-Roses, B.P. 17, 92262, FRANCE \\ ${ }^{2}$ Oak Ridge National Laboratory, Oak Ridge, Tennessee, 37831, P. O. Box 2008, USA \\ ${ }^{3}$ Italian National Agency for New Technologies, Lungotevere Thaon di Revel 76, 00196 Roma, ITALY \\ ${ }^{4}$ Canadian Nuclear Laboratory, 286 Plant Rd., ON K0J 1J0, Chalk River, CANADA \\ ${ }^{5}$ University of Bologna and INFN, Via Irnerio 46, 40126 Bologna, ITALY \\ ${ }^{*}$ Corresponding author. E-mail: luiz.leal@irsn.fr
}

\begin{abstract}
The objective of this paper is to present the results of an evaluation of the gadolinium isotopes with the main focus on the isotopes ${ }^{155} \mathrm{Gd}$ and ${ }^{157} \mathrm{Gd}$. The evaluations were carried out in the resolved resonance region using the Reich-Moore formalism. The originality on the ${ }^{155} \mathrm{Gd}$ and ${ }^{157} \mathrm{Gd}$ evaluations is the addition of new high-resolution capture cross section measurements performed at the neutron time-of-flight, $\mathrm{n}$ - TOF facility for enriched samples and the statistical analysis of the resonance parameters. The resonance analysis was performed with the multilevel R-matrix code SAMMY together with the generalized least-squares technique based on the Bayes' theory.
\end{abstract}

\section{Introduction}

Resolved resonance evaluation of the gadolinium isotopes were carried out using the multilevel R-matrix code SAMMY.[1] Capture measurements for enriched ${ }^{155} \mathrm{Gd}$ and ${ }^{157} \mathrm{Gd}$ isotopes and capture and transmission data for natural gadolinium were used in the evaluation. The natural abundances for the stable gadolinium isotopes range from $0.2 \%$ to $24.84 \%$. The stable isotopes are ${ }^{152} \mathrm{Gd}(0.2 \%),{ }^{154} \mathrm{Gd}(2.18 \%),{ }^{155} \mathrm{Gd}(14.80 \%),{ }^{156} \mathrm{Gd}$ $(20.47 \%),{ }^{157} \mathrm{Gd}(15.65 \%),{ }^{158} \mathrm{Gd}(24.84 \%)$, and ${ }^{160} \mathrm{Gd}$ $(21.86 \%)$, respectively. The isotopes with the highest thermal capture cross sections are ${ }^{155} \mathrm{Gd}$ with thermal cross section of about 65,000 barns and ${ }^{157} \mathrm{Gd}$ which the thermal cross section is of the order of 254,000 barns. Unquestionably, these two isotopes play significant role in criticality safety applications and nuclear reactor technology applications. Consequently, due to their importance a great deal of work has been devoted to the resonance evaluation of these isotopes to extract resonance parameters that described well the experimental data. In addition, detailed statistical analyses of the ${ }^{155} \mathrm{Gd}$ and ${ }^{157} \mathrm{Gd}$ resonance parameters were done on the basis of the s-wave resonances $(l=0)$, since the penetrability for higher angular momentum $(1>0)$ was not significant for the energy region where the resonance analysis was performed. The evaluations presented in this work extend the resolved resonance energy range of the ${ }^{155} \mathrm{Gd}$ and ${ }^{157} \mathrm{Gd}$ isotopes by taking advantage of the high resolution data measurements included in the evaluation. The isotopes ${ }^{156} \mathrm{Gd},{ }^{158} \mathrm{Gd}$ and ${ }^{160} \mathrm{Gd}$ were also reviewed and evaluated with the help of the natural capture cross section and transmission data.
The less abundant isotopes ${ }^{152} \mathrm{Gd}$ and ${ }^{154} \mathrm{Gd}$ were not thoroughly evaluated but just used to complete the full set of resonance parameters in the analysis of the natural samples. Resonance parameters for these isotopes were those listed in the Atlas of Neutron Resonances (ANR).[2] The upper energy of the resonance region for the new gadolinium evaluations is listed in Table 1. Also indicated in Table 1 is the upper resonance energy for the Joint Evaluated Fission and Fusion (JEFF) project, JEFF-3.3 library.

Table 1. Resonance region upper energy

\begin{tabular}{|c|c|c|}
\hline Isotope & $\begin{array}{c}\text { JEFF-3.3 } \\
(\mathrm{eV})\end{array}$ & $\begin{array}{c}\text { New Gd } \\
\text { evaluation } \\
(\mathrm{eV})\end{array}$ \\
\hline${ }^{152} \mathrm{Gd}$ & 2658 & 2658 \\
\hline${ }^{154} \mathrm{Gd}$ & 2760 & 2760 \\
\hline${ }^{155} \mathrm{Gd}$ & 181.8 & 500 \\
\hline${ }^{156} \mathrm{Gd}$ & 1580 & 2250 \\
\hline${ }^{157} \mathrm{Gd}$ & 215 & 500 \\
\hline${ }^{158} \mathrm{Gd}$ & 6037.6 & 10000 \\
\hline${ }^{160} \mathrm{Gd}$ & 2883.7 & 10000 \\
\hline
\end{tabular}

\section{Experimental data}

Transmission and capture data measurements performed at the Gaerttner time-of-flight (TOF) linac facility located at Rensselaer Polytechnic Institute (RPI) [3,4] and data taken at the n_TOF facility [5] were included in the evaluation. The measurements were done at the temperature of $293.6 \mathrm{~K}$. Three transmission data and 
capture yield measurements for natural gadolinium done at RPI were included in the evaluation. In addition, two capture measurements for enriched ${ }^{155} \mathrm{Gd}$ and ${ }^{157} \mathrm{Gd}$ from RPI were also included in the analysis. From n TOF, four measurements of different thicknesses for enriched ${ }^{155} \mathrm{Gd}$ and ${ }^{157} \mathrm{Gd}$ were carefully analyzed. The descriptions of the measured data used in the evaluation are indicated in Table 2. Thermal values and uncertainties indicated in the ANR were also considered in the evaluation.

\section{Method of evaluation}

\subsection{Fitting procedure}

A Reich-Moore [6] resonance analysis and evaluation of the experimental data displayed in Table 2 were carried out with the computer code SAMMY. The experimental data were entered sequentially taking into account the temperature effects, data resolution, normalization, background effects, etc. At each step of the evaluation process an updated resonance parameter and resonance parameter covariance are generated, which are, subsequently feedback into the analysis of the next experimental data in the sequence. This process is repeated several times until a good fit of the experimental data is achieved.

Table 2. Experimental transmission and capture data

\begin{tabular}{|c|c|c|c|c|}
\hline Data Set & $\begin{array}{c}\text { Enrichment } \\
(\%)\end{array}$ & $\begin{array}{c}\text { Energy } \\
\text { Range (eV) }\end{array}$ & $\begin{array}{c}\text { Flight } \\
\text { Path } \\
(\mathrm{m}) \\
\end{array}$ & $\begin{array}{l}\text { Density } \\
(\mathrm{at} / \mathrm{b})\end{array}$ \\
\hline \multicolumn{5}{|c|}{ Natural Gadolinium } \\
\hline $\begin{array}{c}\text { Transmission } \\
\text { (RPI) }\end{array}$ & - & $0.2-300.0$ & 25.585 & $7.806 \times 10^{-4}$ \\
\hline $\begin{array}{c}\text { Transmission } \\
\text { (RPI) }\end{array}$ & - & $0.3-500.0$ & 25.597 & $1.566 \times 10^{-3}$ \\
\hline $\begin{array}{c}\text { Transmission } \\
\text { (RPI) }\end{array}$ & - & $0.3-1000.0$ & 25.597 & $1.566 \times 10^{-3}$ \\
\hline $\begin{array}{c}\text { Capture } \\
\text { (RPI) } \\
\end{array}$ & - & $0.2-1000.0$ & 25.585 & $7.806 \times 10^{-4}$ \\
\hline \multicolumn{5}{|c|}{${ }^{155} \mathbf{G d}$} \\
\hline $\begin{array}{c}\text { Capture } \\
\text { (RPI) } \\
\end{array}$ & 91.74 & $0.2-1000.0$ & 25.567 & $3.083 \times 10^{-4}$ \\
\hline $\begin{array}{c}\text { Capture } \\
\left(\mathrm{n} \_\mathrm{TOF}\right) \\
\end{array}$ & 91.74 & $0.025-50.0$ & 183.90 & $1.236 \times 10^{-6}$ \\
\hline $\begin{array}{l}\text { Capture } \\
\left(\mathrm{n} \_\mathrm{TOF}\right)\end{array}$ & 91.74 & $1.0-1000.0$ & 183.90 & $1.244 \times 10^{-4}$ \\
\hline \multicolumn{5}{|c|}{${ }^{157} \mathrm{Gd}$} \\
\hline $\begin{array}{l}\text { Capture } \\
\text { (RPI) }\end{array}$ & 90.96 & $0.2-1000.0$ & 25.569 & $5.820 \times 10^{-4}$ \\
\hline $\begin{array}{l}\text { Capture } \\
\left(\mathrm{n} \_\mathrm{TOF}\right) \\
\end{array}$ & 88.32 & $0.025-50.0$ & 183.90 & $5.753 \times 10^{-6}$ \\
\hline $\begin{array}{l}\text { Capture } \\
\text { (n_TOF) }\end{array}$ & 88.32 & $1.0-1000.0$ & 183.90 & $2.340 \times 10^{-4}$ \\
\hline
\end{tabular}

An important figure-of-merit that indicates a good fitting of the experimental data is the $\chi^{2}$ provided at the end of each SAMMY run. The resulting set of resonance parameters is expected to reproduce well the experimental data within the quoted experimental uncertainty. SAMMY also generates resonance parameter covariance matrix. The latter is not part of the work presented in this paper. It should be stressed that while it looks a simple matter, the fitting process is, actually a very demanding task. It requires to the evaluator a good perception of the theory as well a decent understanding of the experimental data used in the evaluation. One may derived a set of parameters that fit the data but it can be totally unphysical. Further tests, such as statistical test must be employed to guarantee that the resonance parameters fall close to the underlining physics. The latter issue will be discussed in this paper.

\subsection{SAMMY fitting results}

Comparisons of the SAMMY R matrix fit to the data of the n_TOF capture yield data for the ${ }^{155} \mathrm{Gd}$ and ${ }^{157} \mathrm{Gd}$, in the energy region $0.02 \mathrm{eV}$ to $10 \mathrm{eV}$, are shown in Figures 1. The top curve represents the capture yield for ${ }^{155} \mathrm{Gd}$ and ${ }^{157} \mathrm{Gd}$ comparison is the lower curve.


Fig. 1. Comparisons of SAMMY calculations with the resonance parameter (solid line) of the capture yield of the $\mathrm{n}$ TOF data in the energy region $0.02 \mathrm{eV}$ to $10 \mathrm{eV}$.

Comparison of the SAMMY fitting of the n_TOF and RPI capture yields for ${ }^{155} \mathrm{Gd}$ in the energy $50 \overline{\mathrm{eV}}$ to 250 $\mathrm{eV}$ are shown Figure 2.
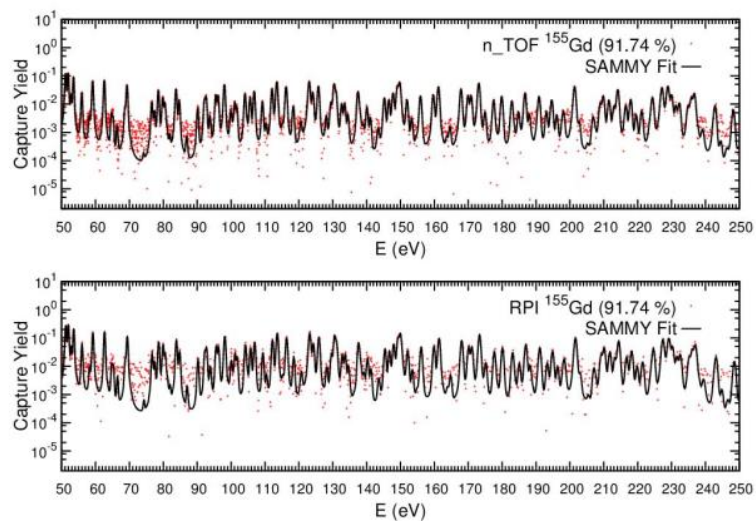

Fig. 2. Comparisons of SAMMY calculations with the resonance parameter (solid line) of the capture yield of the $\mathrm{n}_{-}$TOF and RPI data in the energy region $50 \mathrm{eV}$ to $250 \mathrm{eV}$ for ${ }^{155} \mathrm{Gd}$. 
Similar comparisons for ${ }^{157} \mathrm{Gd}$ are shown in Figure 3.
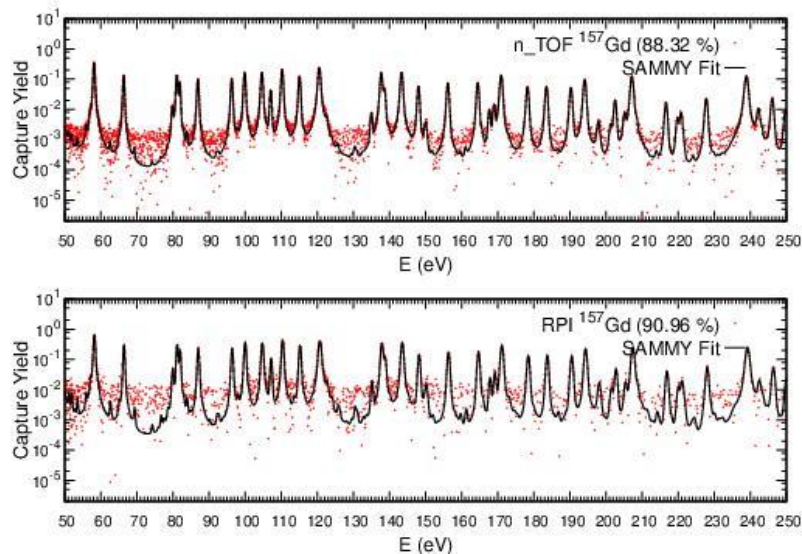

Fig. 3. Comparisons of SAMMY calculations with the resonance parameter (solid line) of the capture yield of the $\mathrm{n} \_$TOF and RPI data in the energy region $50 \mathrm{eV}$ to $250 \mathrm{keV}$ for ${ }^{15 \overline{7}} \mathrm{Gd}$.

Comparisons of the capture yield for the n_TOF and RPI data in the energy range of $250 \mathrm{eV}$ to $5 \overline{0} 0 \mathrm{eV}$ for ${ }^{155} \mathrm{Gd}$ and ${ }^{157} \mathrm{Gd}$ are shown in Figure 4. Results for the fitting of the transmission data for natural gadolinium are shown in Figure 5 in the energy range from $3 \mathrm{eV}$ to 300 $\mathrm{eV}$. The contribution of each gadolinium isotopes is taken in account in the fitting of the transmission data.

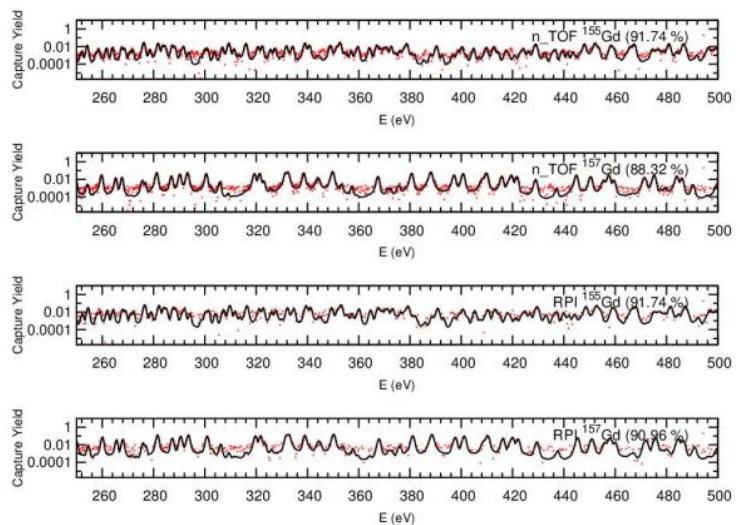

Fig. 4. Comparisons of SAMMY calculations with the resonance parameter (solid line) of the n_TOF and RPI capture yield in the energy range $250 \mathrm{eV}$ to $500 \overline{\mathrm{eV}}$.
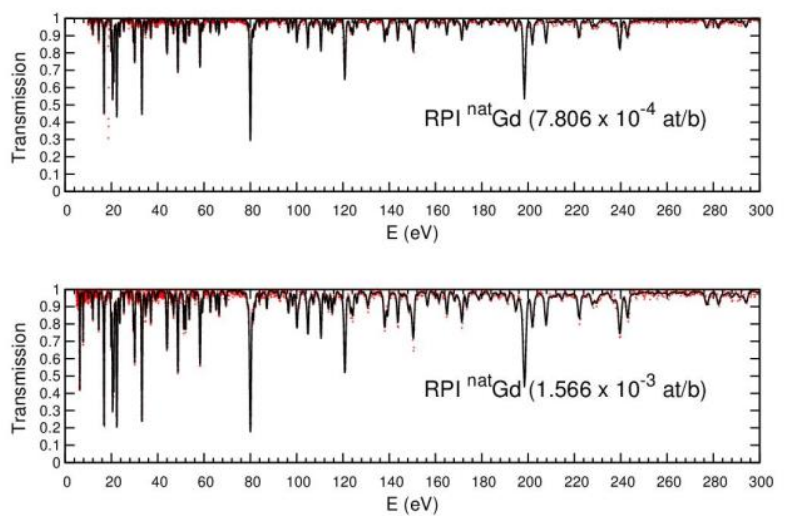

Fig. 5. Comparisons of SAMMY calculations with the resonance parameter (solid line) of the RPI transmission data for natural gadolinium in the energy range $3 \mathrm{eV}$ to $300 \mathrm{eV}$.

Capture and scattering cross section at thermal
$(0.0253 \mathrm{eV})$, capture resonance integral and capture Westcott factor calculated from the resonance parameters obtained in the evaluation for ${ }^{155} \mathrm{Gd},{ }^{156} \mathrm{Gd},{ }^{157} \mathrm{Gd},{ }^{158} \mathrm{Gd}$, and ${ }^{160} \mathrm{Gd}$ are listed in Table 3.

Table 3. Thermal values, resonance integral and Westcott factors

\begin{tabular}{|l|l|l|l|l|l|}
\hline \multirow{2}{*}{ Quantities } & \multicolumn{5}{|c|}{ Gadolinium Isotopes } \\
\cline { 2 - 6 } & ${ }^{155} \mathrm{Gd}$ & ${ }^{156} \mathrm{Gd}$ & ${ }^{157} \mathrm{Gd}$ & ${ }^{158} \mathrm{Gd}$ & ${ }^{160} \mathrm{Gd}$ \\
\hline$\sigma_{s}$ (barns) & 61.58 & 4.88 & 1018.58 & 5.28 & 10.31 \\
\hline$\sigma_{\gamma \text { (barns) }}$ & 61857.42 & 1.93 & 254570.90 & 2.25 & 1.52 \\
\hline$I_{\gamma \text { (barns) }}$ & 1567 & 99.40 & 814.98 & 78.27 & 10.32 \\
\hline$g_{\gamma}$ & 0.83326 & 1.00037 & 0.85204 & 1.00046 & 0.99994 \\
\hline
\end{tabular}

The impact of the values indicated in Table 3 in benchmark calculations are under investigation.

\section{Resonance Parameters Statistics}

Statistical analysis of the s-wave resonance parameters derived in this work for ${ }^{155} \mathrm{Gd}$ and ${ }^{157} \mathrm{Gd}$ were carried out using the SAMDIST [7] component of the SAMMY code. The level spacing distribution and the Dyson-Mehta $\Delta 3$-statistics [8] were carefully examined.

\subsection{Level spacing distribution}

The isotopes ${ }^{155} \mathrm{Gd}$ and ${ }^{157} \mathrm{Gd}$ have identical target spin and parity of $3 / 2^{-}$and consequently the compound nucleus total angular momentum $J^{\pi}$ and parity, for $s$-wave resonances, are $1^{-}$and $2^{-}$, respectively. Assuming a level spacing density proportional to $2 J+1$, the ratio of the average spacing of the two states will be, approximately, $\mathrm{D}_{1}^{-} / \mathrm{D}_{2}^{-} \sim 5 / 3$ which indicates that resonances in the spin state $1^{-}$are less frequent as compared to spin state $2^{-}$. The average level spacing values for each spin and the mixed spin for ${ }^{155} \mathrm{Gd}$ and ${ }^{157} \mathrm{Gd}$ in the energy range from thermal to $500 \mathrm{eV}$ are shown in Table 4.

Table 4. Average level spacing

\begin{tabular}{|c|c|c|}
\hline $\mathrm{D}\left(\mathrm{J}^{\pi}\right)$ & $\begin{array}{c}{ }^{155} \mathrm{Gd} \\
(\mathrm{eV})\end{array}$ & $\begin{array}{c}{ }^{157} \mathrm{Gd} \\
(\mathrm{eV})\end{array}$ \\
\hline $\mathrm{D}\left(1^{-}\right)$ & $5.00 \pm 0.32$ & $15.20 \pm 1.75$ \\
\hline $\mathrm{D}\left(2^{-}\right)$ & $2.46 \pm 0.12$ & $5.99 \pm 0.43$ \\
\hline $\mathrm{D}$ & $1.64 \pm 0.35$ & $4.31 \pm 1.80$ \\
\hline
\end{tabular}

Comparisons of the spacing distributions to the Wigner distributions are shown in Figures 6 and 7 for ${ }^{155} \mathrm{Gd}$ and ${ }^{157} \mathrm{Gd}$, respectively. 

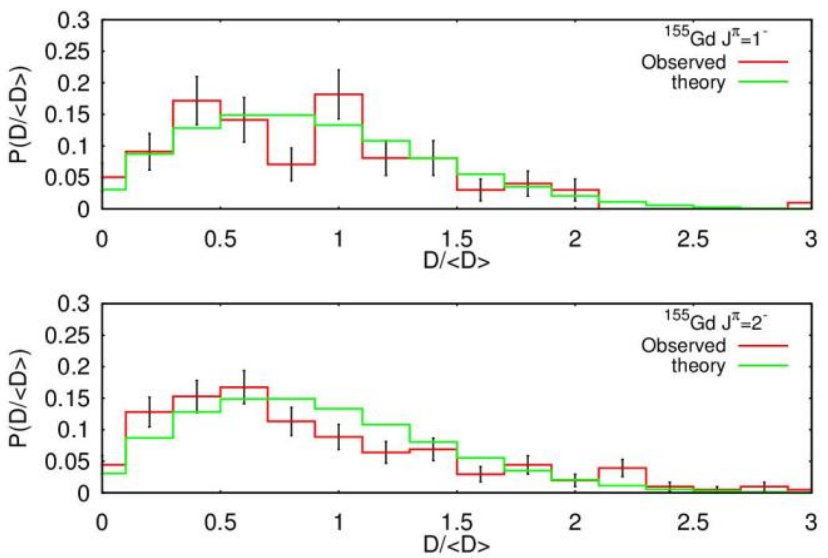

Fig. 6. Wigner distribution (theory) of the resonances spacing in the energy $0 \mathrm{eV}$ to $500 \mathrm{eV}$ for ${ }^{155} \mathrm{Gd}$.
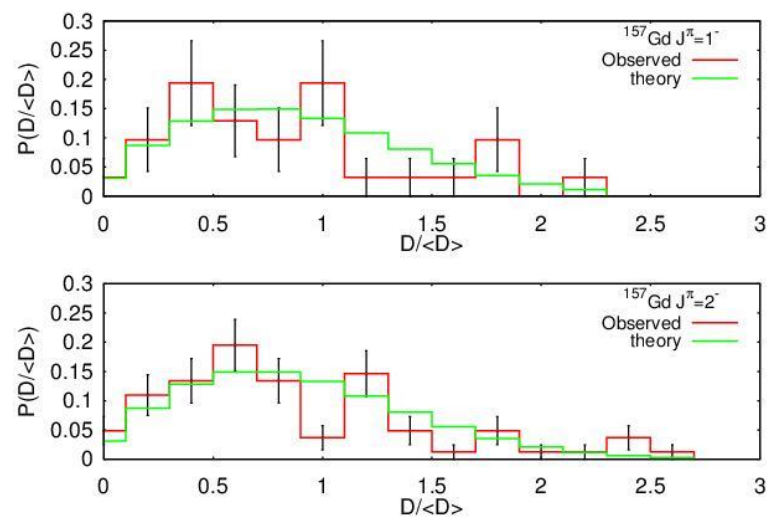

Fig. 7. Wigner distribution (theory) of the resonances spacing in the energy $0 \mathrm{eV}$ to $500 \mathrm{eV}$ for ${ }^{157} \mathrm{Gd}$.

The cumulative number of energy levels for ${ }^{155} \mathrm{Gd}$ and ${ }^{157} \mathrm{Gd}$ in the energy range 0 to $500 \mathrm{eV}$ are shown in Figure 8 and Figure 9, respectively.
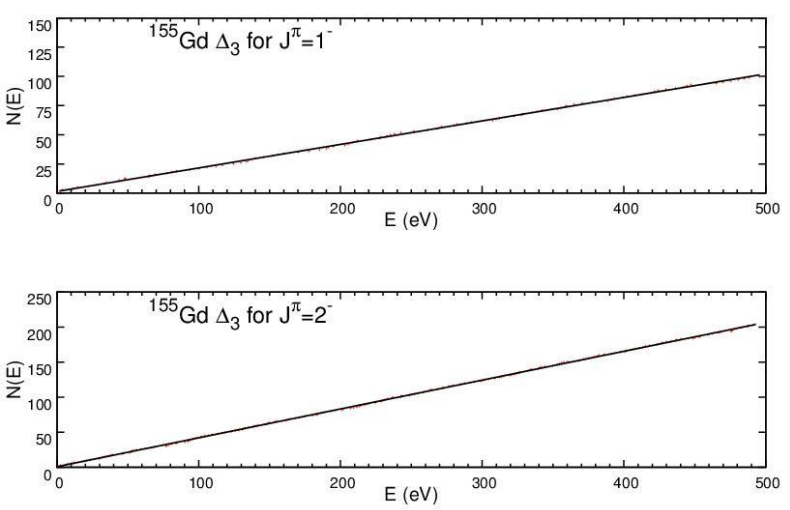

Fig. 8. Cumulative number of energy levels in the energy $0 \mathrm{eV}$ to $500 \mathrm{eV}$ for ${ }^{155} \mathrm{Gd}$.


Fig. 9. Cumulative number of energy levels in the energy $0 \mathrm{eV}$ to $500 \mathrm{eV}$ for ${ }^{157} \mathrm{Gd}$.

The results of the $\Delta_{3}$-statistics tests for ${ }^{155} \mathrm{Gd}$ and ${ }^{157} \mathrm{Gd}$ for the two $\mathrm{J}^{\pi}$ are displayed in Table 5. The $\Delta_{3}$-statistics provides a good insight on the long range correlation between the energy resonance levels, resonance spin assignment, missing levels, etc. It can be noted from Table 5 that in the energy range from 0 to 500 $\mathrm{eV}$ the $\Delta_{3}$-statistics results are in better shape for ${ }^{157} \mathrm{Gd}$ as compared to the results for ${ }^{155} \mathrm{Gd}$. This observation does not come as a surprise since the average resonance spacing for ${ }^{155} \mathrm{Gd}$ is smaller than that for ${ }^{157} \mathrm{Gd}$, that is, more energy levels in ${ }^{155} \mathrm{Gd}$ increases the chance of missing levels. Another observation is that the distribution of levels may not follow strictly a Gaussian orthogonal distribution from which the Wigner distribution is derived but rather a unitary orthogonal distribution. Further tests are under way on this issue.

Table 5. $\Delta 3$-statistics Test

\begin{tabular}{|c|l|c|c|c|}
\cline { 2 - 5 } \multicolumn{1}{c|}{} & \multicolumn{2}{c|}{${ }^{155} \mathrm{Gd}$} & \multicolumn{2}{c|}{${ }^{157} \mathrm{Gd}$} \\
\hline $\mathrm{J}^{\pi}$ & Theory & Experimental & Theory & Experimental \\
\hline $1^{-}$ & $0.459 \pm 0.109$ & 0.744 & $0.344 \pm 0.109$ & 0.519 \\
\hline $2^{-}$ & $0.531 \pm 0.109$ & 0.904 & $0.441 \pm 0.109$ & 0.586 \\
\hline
\end{tabular}

\section{Conclusion and remarks}

This paper presents a new resonance evaluation of the gadolinium isotopes ${ }^{155} \mathrm{Gd},{ }^{156} \mathrm{Gd},{ }^{157} \mathrm{Gd},{ }^{158} \mathrm{Gd}$, and ${ }^{160} \mathrm{Gd}$. Compared to existing evaluations, the resonance energy ranges have been increased on the base of high resolution transmission and capture cross section data. Natural transmission and capture cross section data and capture data for enriched samples of ${ }^{155} \mathrm{Gd}$ and ${ }^{157} \mathrm{Gd}$ have been used in the analysis evaluation. New capture cross section values at thermal energy have been proposed. The impact of the new evaluation on benchmark calculation and results is presently under study. Not described in this work nevertheless resonance parameter covariance for the evaluated isotopes have also been derived. 


\section{REFERENCES}

[1] N. M. LARSON, "Updated Users' Guide for SAMMY: Multilevel R-Matrix Fits to Neutron Data Using Bayes' Equations," ORNL/TM-9179/R8 (ENDF-364/R2) (2008).

[2] S. F. Mughabghab, "Atlas of Neutron Resonances Resonance Parameters and Thermal Cross Sections $Z=1-100, " 5^{\text {th }}$ Edition, National Nuclear Data Center, Brookhaven National Laboratory, Upton, USA (2006).

[3] Y. R. Kang, M. W. Lee, G. N. Kim, T. I. Ro, Y. Danon, D. Williams, G. Leinweber, R. C. Block, D. P. Barry, and M. J. Rapp, "Neutron Capture Measurements and Resonace Parameters of Gadolinium," Nucl. Sci. Eng. 180, 86-116(2015).

[4] G. Leinber, E. P. Barry, M. J. Trbovich, J. A. Burke, N. J. Drindak, H. D. Knox, R. V. Ballad, R. C. Block, Y. Danon, and L. I. Severnyak, "Neutron Capture and Total Cross-Section Measurements and Resonance Parameters of Gadolinium,” Nucl. Sci. Eng. 154, 261-279(2006).

[5] M. Mastromarco, A. Manna, O. Aberle, J. Andrzejewski, L. Audouin, M. Bacak, J. Balibrea, M. Barbagallo, F. Bečvár, E. Berthoumieux, J. Billowes, D. Bosnar, A. Brown, M. Caamaño, F. Calviño, M. Calviani, D. Cano-Ott, R. Cardella, A. Casanovas, D.M. Castelluccio, F. Cerutti, Y. H. Chen, E. Chiaveri, G. Clai, N. Colonna, G. Cortés, M.A. Cortés-Giraldo, L. Cosentino, L. A. Damone, M. Diakaki, C. Domingo-Pardo, R. Dressler, E. Dupont, I. Durán14, B. Fernández-Domínguez, A. Ferrari, P. Ferreira, P. Finocchiaro, V. Furman, K. Göbel, A. R. García, A. Gawlik, S. Gilardoni, T. Glodariu, I. F. Gonçalves, E. González-Romero, E. Griesmayer, C. Guerrero, F. Gunsing, A. Guglielmelli, H. Harada, S. Heinitz, J. Heyse, D. G. Jenkins, E. Jericha, F. Käppeler, Y. Kadi, A. Kalamara, P. Kavrigin, A. Kimura, N. Kivel, I. Knapova, M. Kokkoris, M. Krtička, D. Kurtulgil, E. Leal-Cidoncha, C. Lederer, H. Leeb, J. Lerendegui-Marco, S. J. Lonsdale, D. Macina, J. Marganiec, T. Martínez, A. Masi, C. Massimi, P. Mastinu, E. A. Maugeri, A. Mazzone, E. Mendoza, A. Mengoni1, P. M. Milazzo, F. Mingrone, A. Musumarra, A. Negret, R. Nolte, A. Oprea, N. Patronis, A. Pavlik, J. Perkowski, I. Porras, J. Praena, J. M. Quesada, D. Radeck, T. Rauscher, R. Reifarth, F. Rocchi, C. Rubbia, J.A. Ryan, M. Sabaté-Gilarte, A. Saxena, P. Schillebeeckx, D. Schumann, P. Sedyshev, A. G. Smith, N. V. Sosnin, A. Stamatopoulos, G. Tagliente, J. L. Tain, A. Tarifeño-Saldivia, L. Tassan-Got, S. Valenta, G. Vannini, V. Variale, P. Vaz, A. Ventura, V. Vlachoudis, R. Vlastou, A. Wallner, S. Warren, C. Weiss, R. Winants, P. J. Woods, T. Wright, and P. Žugec, "Cross section measurements of $155,157 \mathrm{Gd}(\mathrm{n}, \gamma)$ induced by thermal and epithermal neutrons," Eur. Phys. J. A (2019) 55: 9

[6] C. W. Reich and M. S. Moore, "Multilevel Formula for the Fission Process," Phys. Rev. 111, 192(1958).

[7] L. C. Leal and N. M. Larson, "SAMDIST: A Computer Code for Calculating Statistical Distributions for R-Matrix Resonance Parameters, ORNL/TM-13092, September 1995.

[8] F. J. Dyson and M. L. Mehta J. Lath. Phys. 104, 483 (1956). 